\title{
Entwicklung und Evaluierung eines Algorithmus zur automatisierten Rekonfiguration von Niederspannungsnetzen
}

\author{
D. Herbst@, R. Schürhuber, S. Henein, P. Zehetbauer, D. Fellner, A. Einfalt, E. Schmautzer, L. Fickert
}

\begin{abstract}
Aktuelle Trends, wie die zunehmende Verbreitung von Ladestationen für Elektrofahrzeuge (EVCSs) mit und ohne Batteriespeicher in Kombination mit Wärmepumpen (WP) und Klimaanlagen, stellen Niederspannungsnetze (NS-Netze) bzW. deren Betrieb vor neuartige Herausforderungen. Diese können beispielsweise in Form von nennenswerten Lastfluss- sowie Kurzschlussproblemen in deren traditionellen, meist radialen Strukturen bzw. Topologien auftreten. Ein wesentliches Beispiel dafür ist die Überlastung von Leitungssegmenten durch Lasten mit erheblicher Gleichzeitigkeit (EVCSs, WPs, Klimaanlagen etc.). Darüber hinaus können hohe lokale Einspeisungen durch dezentrale Stromerzeuger (z. B. Photovoltaikanlagen, Kleinwasserkraftwerke, Batterie-Energiespeichersysteme) zu Problemen der Spannungsqualität (bspw. Verletzung der Spannungsgrenzen, Spannungsabfall, schnelle Spannungsänderungen) führen. Das Projekt "Power System Cognification“ (PoSyCo) entwickelt Lösungsansätze, um diese eben genannten Herausforderungen zu bewältigen. Es zielt darauf ab, ein "SOFTprotection"-System zu implementieren, das zur Vermeidung von systemkritischen Zuständen des Niederspannungsnetzes bzW. von Fehlern beiträgt und als Ergänzung zur herkömmlichen „HARDprotection“ (u. a. Sicherungen, Leistungsschalter) dienen soll. Im Zuge dieses Beitrags wird einer der dafür entwickelten Methoden in Form eines Algorithmus zur automatisierten Netzrekonfiguration auf Ebene der Niederspannung (NS) näher beschrieben.
\end{abstract}

Schlüsselwörter: Niederspannungsnetz; Schutz; automatisierte Netzrekonfiguration; temporäre Vermaschung; Leitungsüberlastungen; Trennstellenverlegung

\section{Development and evaluation of an algorithm for the automated reconfiguration of low-voltage grids.}

Today's trends, like the increasing use of electric vehicle charging stations (EVCSS) with and without battery storage in combination with heat pumps (HPS) and air conditioning systems, pose new challenges to low-voltage grids (LV grids) and their operation. These can occur, for example, in the form of significant load flow and short-circuit problems in their traditional, mostly radial structures or topologies. An important example is the overloading of line segments through loads with significant simultaneity (EVCSS, HPS, air conditioning systems, etc.). In addition, high local feed-ins from decentralized power generators (e.g., photovoltaic systems, small hydropower plants, battery energy storage systems) can lead to power quality problems (e.g., violation of voltage limits, voltage drop, rapid voltage changes). The project "Power System Cognification" (PoSyCo) develops solutions to tackle these challenges. It aims to implement a "SOFTprotection" system that helps avoid system-critical states of the low-voltage grid or faults and is intended to supplement conventional "HARDprotection" (e.g., fuses, circuit breakers). In the course of this paper, one of the methods developed for this purpose is described in more detail in the form of an algorithm for automated grid reconfiguration at the low-voltage (LV) level.

Keywords: low-voltage grid; protection; automated grid reconfiguration; temporary meshing; line overloading; separation point relocation

Eingegangen am 12. Juli 2021, angenommen am 17. September 2021, online publiziert am 4. Oktober 2021 (c) The Author(s) 2021

\section{Einleitung}

Niederspannungsverteilnetze bilden die Grundlage für die elektrische Energieversorgung einer großen Anzahl von Kundenanlagen im Haushalts- und Gewerbebereich. Unter anderem aufgrund klimapolitischer Ziele und Verpflichtungen wird der Ausbau erneuerbarer Energiequellen (renewable energy sources, RES) vorangetrieben [1]. Neben den bereits weit verbreiteten Großprojekten werden immer mehr dezentrale Anlagen zur Erzeugung und Speicherung von elektrischer Energie realisiert. Darüber hinaus gibt es Regularien (z. B. [2]) im Zusammenhang mit der flächendeckenden Ausrollung der Elektromobilität, insbesondere batterieelektrischer Fahrzeuge (battery electric vehicles, BEVs), welche wiederum zu einem rasanten Wachstum der benötigten Ladeinfrastruktur führen. Ergänzend dazu ist - Dank aktueller Entwicklungen - ein Anstieg an wei-

Herbst, Daniel, Institut für Elektrische Anlagen und Netze, Technische Universität Graz, Inffeldgasse 18/1, 8010 Graz, Österreich (E-Mail: daniel.herbst@tugraz.at); Schürhuber, Robert, Institut für Elektrische Anlagen und Netze, Technische Universität Graz, Graz, Österreich; Henein, Sawsan, Center for Energy, AIT Austrian Institute of Technology $\mathrm{GmbH}$, Wien, Österreich; Zehetbauer, Paul, Center for Energy, AIT Austrian Institute of Technology GmbH, Wien, Österreich; Fellner, David, Center for Energy, AIT Austrian Institute of Technology GmbH, Wien, Österreich; Einfalt, Alfred, CT RDA IOT - Siemens AG Österreich, Wien, Österreich; Schmautzer, Ernst, Institut für Elektrische Anlagen und Netze, Technische Universität Graz, Graz, Österreich; Fickert, Lothar, Institut für Elektrische Anlagen und Netze, Technische Universität Graz, Graz, Österreich 
teren leistungsstarken Verbrauchern wie beispielsweise Klimaanlagen oder Wärmepumpen auf Ebene der Niederspannung (NS) erkennbar. Dezentrale Erzeugungseinheiten basierend auf erneuerbaren Energiequellen (z. B. Photovoltaikanlagen) sowie Ladestationen für Elektrofahrzeuge (electric vehicle charging stations, EVCSs) und die genannten leistungsstarken Verbraucher sind in hohem Maße in das Niederspannungsverteilernetz integriert. Auch deuten die aktuellen Entwicklungen in der Branche auf einen mittelfristigen Anstieg an in das NS-Netz zu integrierende Prosumer wie bspw. Batterieenergiespeichersysteme (battery energy storage systems, BESSs) oder auf sogenannte Vehicle-to-Grid (V2G) Technologien, wodurch Traktionsbatterien von Elektrofahrzeugen netzdienlich (z. B. zu Zwecken der Netzstützung in Spitzenlastzeiten) verwendet werden könnten. Daraus ergeben sich zunehmende Herausforderungen für das Niederspannungsnetz, vor allem aufgrund der Bidirektionalität und der aus dem Leistungsanstieg resultierenden Strom- bzw. Lastflüsse. Je nach nationaler Umsetzung der entsprechenden Richtlinien der Europäischen Union (EU) (u.a. [3]) kann die Bildung von regionalen oder lokalen Energiegemeinschaften zudem noch zur Verstärkung dieser Entwicklung führen. Historische lastdominierte Planungskriterien verlieren ihre Gültigkeit und es besteht - aus wirtschaftlichen Gründen - der Wunsch, Niederspannungsnetze näher an ihren natürlichen Grenzen zu betreiben. Im Rahmen des Projekts PoSyCo (Power System Cognification) analysiert ein breit aufgestelltes Projektkonsortium aus Forschung und Industrie gemeinsam mit einem Verteilnetzbetreiber (VNB) diese neuen Anforderungen aus unterschiedlichen Perspektiven. Dabei werden innovative Lösungen entwickelt, um neuartige Netzbetriebskonzepte unter Berücksichtigung der langfristigen Gewährleistung des Personen- und Anlagenschutzes sowie der Versorgungssicherheit zu ermöglichen und somit den Übergang von einem konventionellen Niederspannungsnetz zu einem flexibleren Smart Grid sicherzustellen. PoSyCo zielt darauf ab, ein sogenanntes "SOFTprotection"-System zu implementieren, das zur Vermeidung von Fehlern bzw. Störungen im Netz beiträgt und als Ergänzung zur konventionellen „HARDprotection" (Sicherungen, Leistungsschalter) dient. Dafür wurden im Zuge des Forschungsprojekts sechs Use Cases (UCs) definiert, um die genannten Herausforderungen mittels unterschiedlicher Ansätze bzw. Methoden zu bewältigen. Dieser Beitrag fokussiert sich dabei auf PoSyCos UC4 zum Thema "Überlastschutz durch temporäre Vermaschung" und stellt den dafür entwickelten Algorithmus sowie erste Test- und Simulationsergebnisse vor. Abgerundet werden die Ergebnisse durch eine Sensitivitätsanalyse der Gewichtungsfaktoren der zur Bewertung der verschiedenen Netzkonfigurationen errechneten Kennzahlen (key performance indicators, KPIs).

\section{Methodik}

\subsection{Use Cases des Forschungsprojekts PoSyCo}

In der Projekteingangsphase wurden in Anlehnung an den Standard IEEE 29148:2018 [4] sechs Use Cases zur Umsetzung des in der Einleitung beschriebenen SOFTprotection-Konzepts definiert und ausgearbeitet. Abbildung 1 zeigt eine Übersicht der Use Cases 0 bis 5 sowie deren Einbettung in die Physikalische, die Informations- und Kommunikationstechnik (IKT) sowie die Prozess Dimension beziehungsweise in die drei Stufen Aggregation, Aggregation \& Aktion und Aggregation \& Aktion \& Adaption. Die erarbeiteten UCBeschreibungen $[5,6]$ bilden die Grundlage für die Entwicklung von Methoden und Algorithmen zur Realisierung des SOFTprotectionSystems.

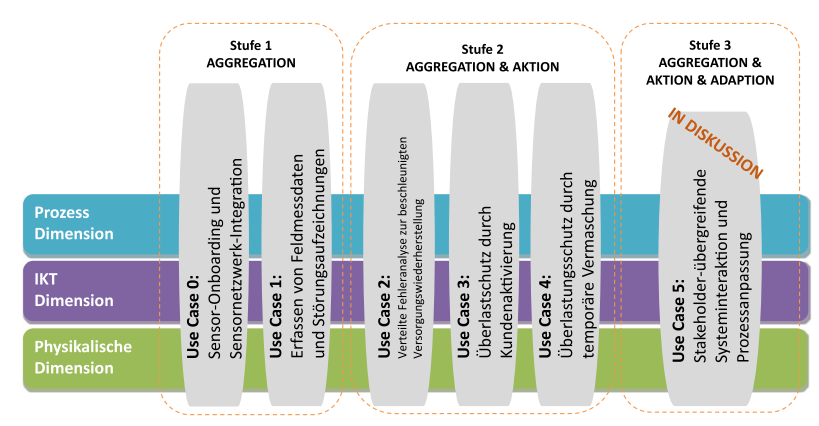

Abb. 1. Übersicht der PoSyCo Use Cases [6]

Dieses Paper konzentriert sich auf UC4, welcher den Einsatz von automatisierten, ferngesteuerten Schaltgeräten (z. B. Leistungsschaltern) untersucht, um mögliche lokale Überlastungen von Leitungsabschnitten durch temporäre Vermaschung bzw. automatisierte Rekonfiguration von NS-Teilnetzen zu verhindern, siehe auch [7-10].

\subsection{Methoden und Ansätze}

Basierend auf den UCs 1 und 2 werden zugehörige Methoden bzw. Konzepte zur Erkennung von herannahenden Überlastsituationen und Fehlern entwickelt. Dazu werden die vom SOFTprotectionSystem aggregierten Daten nach bestimmten Kriterien ausgewertet. Beispiele hierfür sind die ortsabhängige Verletzung des Spannungsbandes $\left(U<U_{\min }\right.$ oder $\left.U>U_{\max }\right)$ oder eines entsprechenden Überstromwertes $\left(I>I_{\max }\right)$ an bestimmten Leitungen über einen vordefinierten Zeitraum.

Nach Erkennen eines nicht betriebskonformen Zustandes wird das betroffene NS-Teilnetz detailliert untersucht. Zunächst wird anhand einer Lastflussberechnung des Teilnetzes entschieden, ob weitere Maßnahmen erforderlich sind. Ergänzend zur Lastflussberechnung kann auch eine Zustandsschätzung (en. State Estimation) des Netzes eingesetzt werden, um aufgrund der zurzeit unvollständigen verfügbaren Messdaten im Niederspannungsnetz ein stabiles Abbild des Lastflusses zu generieren.

Ist dies der Fall, beispielsweise aufgrund einer Überlastung einzelner Betriebsmittel, muss eine erfolgversprechende Maßnahme gewählt werden, um in den unkritischen (normalen) Betrieb zurückzukehren. Dies kann durch eine der folgenden Maßnahmen bzw. Lösungen unter entsprechenden Voraussetzungen (z. B. Vorhandensein von ausreichend dimensionierten Leitungen inkl. fernsteuerbarer Schutz- bzw. Schaltelemente, entsprechende Netztopologien, steuerbare Kundenanlagen) erreicht werden:

- Netzdienliches Verhalten von Kundenanlagen, z. B. gesteuerte EVCS, Batterie-Energiespeichersysteme oder Wärmepumpen (WP)

- Temporäre Vermaschung bzw. Rekonfiguration einzelner Abschnitte von Niederspannungsnetzen, um einen gleichmäßigeren Lastfluss zu erreichen oder Leitungen unter der Voraussetzung, dass die Funktion des Schutzes entsprechend berücksichtigt wird [7-11], zu entlasten.

Die beiden, in der Auflistung dargestellten Maßnahmen beschreiben vorrangig die Auswahl, welche im Zuge des Forschungsprojekts PoSyCo betrachtet werden. Weitere mögliche Ansätze zur Rückkehr zum Normalbetrieb können beispielsweise noch die temporäre Nutzung einzelner anderer Abschnitte oder Komponenten des NSNetzes unter geeigneten Bedingungen (z. B. geplante Reservekapazitäten) oder der Einsatz von regelbaren Ortsnetztransformatorstationen (rONTs) darstellen. 


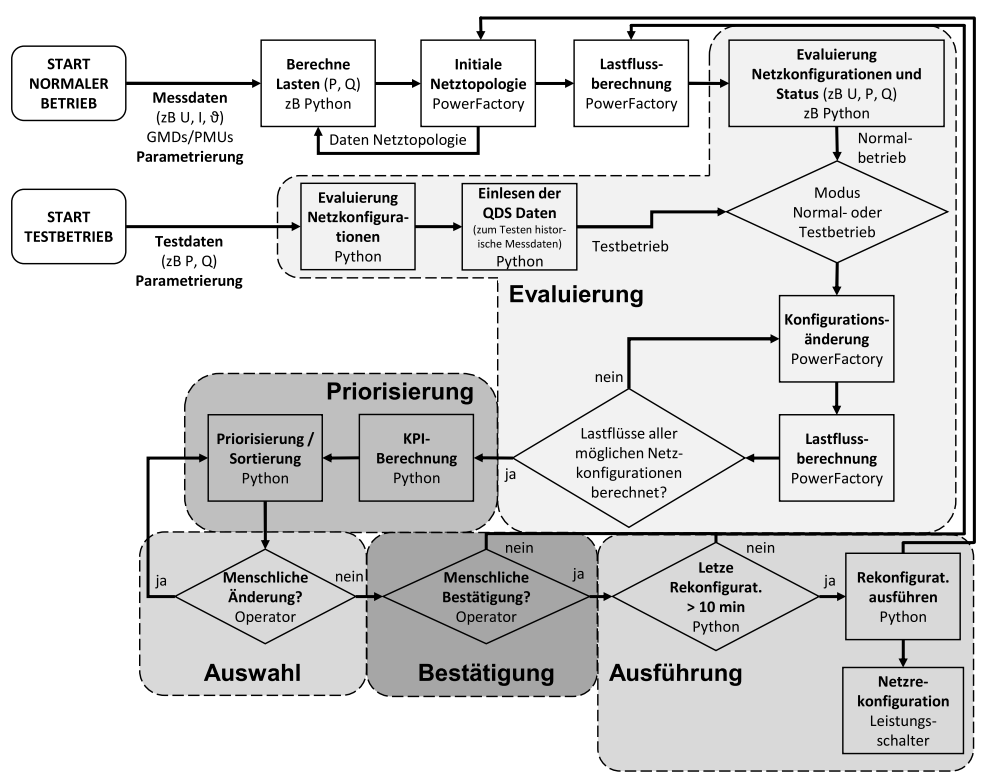

Abb. 2. Blockdiagramm der Entwicklungsumgebung samt dem SMM Algorithmus [8, 10, 12]

\section{Entwickeltes Konzept}

Moderne Niederspannungsnetze sind in hohem Maße mit dezentralen erneuerbaren Energiequellen wie PV-Anlagen in Kombination mit Ladestationen für Elektrofahrzeuge, Wärmepumpen oder Klimaanlagen mit hoher Leistung durchsetzt. Beispielsweise kann nun die dezentrale Einspeisung in einem Knoten in Verbindung mit einem hohen Leistungsbedarf in einem benachbarten Knoten zu Überlastsituationen in einzelnen Leitungsabschnitten zwischen den Knoten führen welche nicht unbedingt von den primären Schutzeinrichtungen (z. B. Sicherungen, Leistungsschalter) am Verteiltransformator erkannt werden. Eine mögliche Lösung, um solche Überlasterscheinungen von NS-Leitungen zu verhindern, ist die bereits erwähnte temporäre Vermaschung bzw. Rekonfiguration.

\subsection{Switching Management Module (SMM)}

Zur Umsetzung des Ansatzes der temporären Vermaschung wird ein spezielles Verfahren zur automatisierten Netzrekonfiguration entwickelt. Den Kern des Konzepts bildet das sogenannte Switching Management Module (SMM) welches aus den folgenden fünf Teilmodulen besteht:

- Evaluierung der (aufgrund der verfügbaren Topologie) möglichen Netzkonfigurationen,

- Priorisierung von möglichen Netzkonfigurationen,

- Auswahl der bevorzugten Konfiguration,

- Bestätigung der ausgewählten Netzkonfiguration und

- Ausführung der Rekonfiguration.

Abbildung 2 zeigt das Blockdiagramm der designten Entwicklungsumgebung samt dem darin eingebetteten SMM Algorithmus und den notwendigen Komponenten. Weiters wird eine Unterscheidung zwischen normalem (realen) Betrieb und Testbetrieb vorgenommen. Die nachfolgenden Unterkapitel beschreiben die Initialisierung (Voreinstellungen, Parameter) des Systems sowie die fünf genannten Teilmodule des SMM-Algorithmus hinsichtlich ihrer Reihenfolge und ihrer Funktionalität.

In diesem Beitrag wird nur der Testbetrieb dargestellt. Zudem wurde am Anfang des Forschungsprojekts PoSyCo entschieden, aus
Gründen der Reproduzierbarkeit der Ergebnisse und zur Entwicklung der Modelle sowie Lösungsalgorithmen, vorerst im Testbetrieb nicht explizit mit State Estimation zu arbeiten. Darüber hinaus ist der Einsatz von State Estimation im normalen Betrieb allerdings geplant. Bei diesem Betriebszustand wird davon ausgegangen, dass sämtliche Messdaten beispielsweise von Smart Metern oder ähnlichen Betriebsmitteln als 5- bis 15-Minuten Messwerte vorliegen was eine für den Algorithmus ausreichende Rechenzeit gewährleistet.

\subsubsection{Parameter und Voreinstellungen}

Nach dem ersten Start des SMM werden die gesamten Parameter und Voreinstellungen, die für den Betrieb des Systems notwendig sind, initialisiert. Der dafür erforderliche Datensatz enthält Basisbzw. Standardeinstellungen wie beispielsweise für die Rekonfiguration empfohlene Trigger- bzw. Schwellenwerte (z. B. Spannung, Strom, Leistung, $\cos (\varphi)$, Temperatur).

Die nachfolgenden fünf Submodule werden nach Start des Systems sowie nach einmaliger Initialisierung kontinuierlich in Schleifenform abgearbeitet.

\subsubsection{Evaluierung}

Basierend auf der gesamten bestehenden Netztopologie (digitales Abbild des betrachteten NS-Teilnetzes respektive der betrachteten Ortsnetzstation inkl. Transformatoren, Leitungen, Schaltelementen bzw. Trennstellen und Lasten) evaluiert das SMM alle möglichen Netzkonfigurationen und berechnet die zugehörigen Lastflüsse. Die zugehörigen Lastflussberechnungen werden für Entwicklungs- bzw. Testzwecke auf tatsächliche historische Messdaten oder auf generierte synthetische Datensätze bezogen. Dabei wird zur Ermittlung des Worst Case die Lastverteilung im Netz zu jenem Zeitpunkt herangezogen, an dem die maximale Auslastung der - bezogen auf den gewählten Betrachtungszeitraum (z. B. ein volles Jahr) - am häufigsten ausgelasteten Leitung auftritt. Dies wird anhand des folgenden Ablaufs durchgeführt:

a) Verfügbarkeitsprüfung der installierten Geräte, um eine Netzrekonfiguration durchzuführen (z. B. verfügbare Leitungen oder Leistungsschalter bzw. Trennstellen) 
Tab. 1. Verwendete Formelzeichen zu den KPI-Gleichungen

\begin{tabular}{ll}
$L S$ & Leistungsschalter \\
$k_{m}$ & Gewichtungsfaktor für jeden $\mathrm{KPI}_{m}$ mit $m \in\{1,2, \ldots, 6\}$ \\
$L_{1}$ & Leitung \\
$n_{L}$ & Anzahl an Leitungen \\
$n_{T}$ & Anzahl an Sammelschienen/Terminals \\
$n_{v}$ & Anzahl an Variationen bzw. Netzkonfigurationen \\
$T_{i}$ & Sammelschienen/Terminal \\
$u$ util & Leitungsauslastung (en. line utilisation) \\
$V_{j}$ & Variation, Netzkonfigurationen \\
$V_{\max }$ & Obere Spannungsgrenze für $V_{T_{i}}$ \\
$V_{\min }$ & Untere Spannungsgrenze für $V_{T_{i}}$ \\
$V_{T_{i}}$ & Spannung am Terminal $T_{i}(3-$ ph. RMS) \\
\hline &
\end{tabular}

b) Evaluierung möglicher Netzkonfigurationen (binäre Variation aller vorhandenen Schaltelemente)

c) Lastflussberechnung der evaluierten Netzkonfigurationen.

Die für die Worst-Case-Ermittlung erforderliche quasi-dynamische Simulation (QDS) zur Vorbereitung der Basisdaten wird in diesem Beitrag nicht näher betrachtet.

\subsubsection{Priorisierung}

Nach der Evaluierung sämtlicher möglicher Netzkonfigurationen und deren zugehöriger Lastflussberechnungen basierend auf den erwähnten QDS-Ergebnissen erfolgt die Priorisierung bzw. Sortierung der einzelnen Konfigurationen gemäß der eingangs definierten Voreinstellungen. Dafür werden basierend auf den Ergebnissen der QDS sowie der Lastflüsse entsprechende Kennzahlen (key performance indicators, KPIs) für jede mögliche Netzkonfiguration berechnet. Nachfolgend werden die dafür entwickelten sechs KPIs zusammengestellt und rudimentär beschrieben, die dabei verwendeten Abkürzungen sind Tab. 1 zu entnehmen. $[8,10,12]$

- $\mathrm{KPl}_{1}$ : Verletzung des Spannungsbandes - Anzahl der Verletzung der Spannungsgrenzen normiert auf die höchste auftretende Anzahl von Verletzungen bezogen auf alle Netzkonfigurationen.

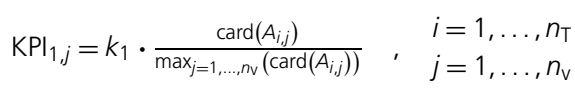

mit card $\left(A_{i, j}\right) \ldots$ Kardinalität/Anzahl der Elemente von $A_{i, j}$ und

$$
A_{i, j}=\left\{T_{i} \mid V_{T_{i}}<V_{\min } \vee V_{T_{i}}>V_{\max }\right\}
$$

- $\mathrm{KPI}_{2}$ : Verletzung der maximal zulässigen Auslastung einer Leitung - Anzahl der überlasteten Leitungen, normiert auf die höchste auftretende Anzahl an Verletzungen der Auslastungsgrenzen bezogen auf alle Netzkonfigurationen.

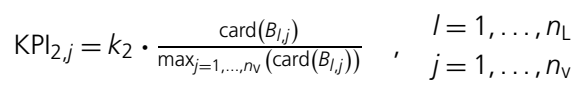

mit card $\left(B_{l, j}\right) \ldots$ Kardinalität/Anzahl der Elemente von $B_{l, j}$ und

$$
B_{l^{\prime}}=\left\{L_{l} \mid u t i l_{L_{l}}>\text { util } I_{\max }\right\}
$$

- $\mathrm{KPI}_{3}$ : Gesamtverluste des Netzabschnittes - Wert der Gesamtnetzverluste des entsprechenden NS-Teilnetzes, normiert auf die maximal auftretenden Gesamtnetzverluste bezogen auf alle Netzkonfigurationen.

$$
\mathrm{KPI}_{3, j}=k_{3} \cdot \frac{S_{\text {Netzverluste }, j}}{\max _{j=1, \ldots, n_{\mathrm{v}}}\left(S_{\text {Netzverluste }, j}\right)}
$$

- $\mathrm{KPI}_{4}$ : Reduktion der Auslastung von Leitungen - Entlastung der ursprünglich am stärksten belasteten Leitung (gemäß dem QDSErgebnis der Ausgangsnetzkonfiguration), normiert auf die maximal auftretende Leitungsbelastung bezogen auf alle Netzkonfigurationen.

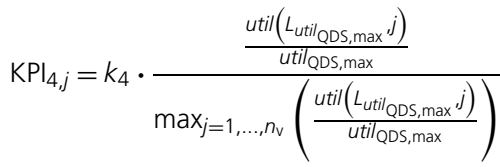

- $\mathrm{KPI}_{5}$ : Distanz ausgelastete Leitung zu Schaltelement - Abstand zwischen der ursprünglich am stärksten ausgelasteten Leitung (gemäß dem QDS-Ergebnis der Ausgangsnetzkonfiguration) und dem betroffenen Schaltelement. Aufgrund der Tatsache, dass je nach Rekonfiguration ein oder mehrere Schaltelemente in Frage kommen können wird immer die kürzeste elektrische Distanz herangezogen.

$$
\mathrm{KPI}_{5, j}=k_{5} \cdot \frac{\min \left(\operatorname{Abstand}\left(L S, L_{\text {util QDS, max }}\right)_{j}\right)}{\max _{j=1, \ldots, n_{v}}\left(\min \left(\operatorname{Abstand}\left(L S, L_{\text {util QDS, } \max }\right)_{j}\right)\right)}
$$

- $\mathrm{KPI}_{6}$ : Vermaschung des Netzabschnittes - Binärer Wert zur Kennzeichnung, ob das Teilnetz radial oder vermascht ist. [15]

$$
\mathrm{KPI}_{6, j}=k_{6} \cdot\left\{\begin{array}{ccc}
1 & \text { wenn } & V_{j} \text { vermascht } \\
0 & \text { wenn } & V_{j} \text { radial }
\end{array}\right.
$$

\subsubsection{Auswahl}

Basierend auf der berechneten Priorisierung ist eine bestimmte Netzkonfiguration auf Grundlage zweier prinzipieller Möglichkeiten auszuwählen:

- Automatische Auswahl einer Netzkonfiguration basierend auf der höchsten, vom SMM automatisiert ermittelten, Priorität.

- Manuelle Auswahl einer Netzkonfiguration, welche mit Expert*innenwissen über Arbeitsprozesse und -abläufe bestimmt wird, z. B. aufgrund geplanter bzw. anstehender Wartungsarbeiten oder anderer Ursachen.

\subsubsection{Bestätigung}

Zur Ausführung der automatisierten Rekonfiguration ist die Möglichkeit vorzusehen, dass die zugehörige Schalthandlung durch eine verantwortliche Person (z. B. ein Operator) seitens Netzbetreiber*in bestätigt werden kann.

\subsubsection{Ausführung}

Nach der Bestätigung durch die verantwortliche Person wird das betroffene Niederspannungsteilnetz durch das SMM bzw. das SOFTprotection-System automatisiert rekonfiguriert indem spezifische Schaltbefehle an den/die betroffenen Leistungsschalter gesendet werden.

Wesentliche Voraussetzungen für ein solches System bzw. die damit verbundene automatisierte Rekonfiguration eines Niederspannungsnetzes liegen einerseits in der Verfügbarkeit von ausreichend Messdaten bzw. Möglichkeiten des Monitorings und andererseits im Vorhandensein von fernbedienbaren Schaltelementen wie beispielsweise Leistungsschaltern. Zudem sei hier erwähnt, dass im Falle von automatisierten Rekonfigurationen (beispielsweise im Sinne von Trennstellenverlegungen) entsprechende make-before- 


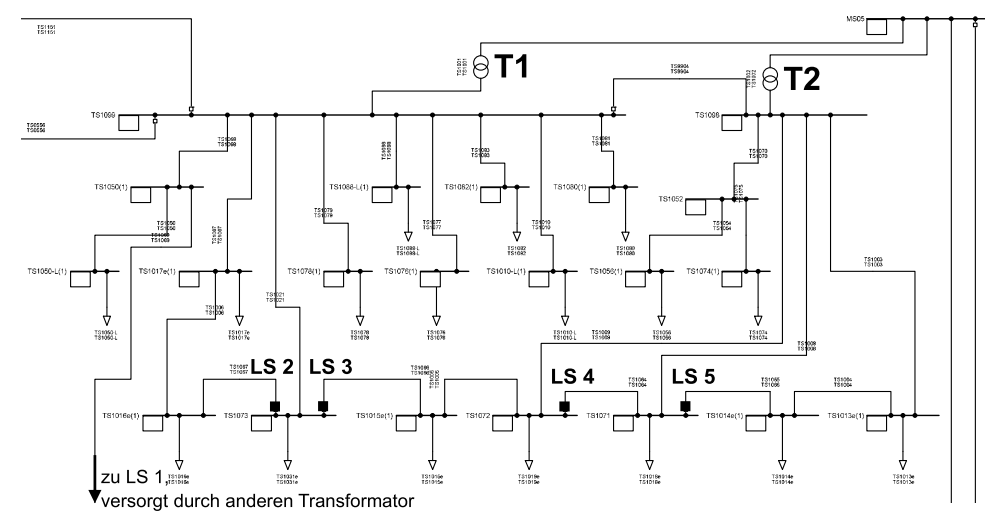

Abb. 3. Urbanes Niederspannungstestnetz aspern Seestadt (Teilnetz TS10) samt den beiden Transformatoren T1 und T2 und den Leistungsschaltern LS 2 bis LS $5[8,10,11]$

break-Routinen zu implementieren sind, um die unterbrechungsfreie Versorgung sämtlicher Kundenanlagen zu gewährleisten.

\section{Simulationen und Ergebnisse}

In einem ersten Schritt wird der entwickelte SMM-Algorithmus mit verschiedenen NS-Testnetzen für unterschiedliche Szenarien getestet. Die dahingehend verwendeten digitalen Abbilder realer Niederspannungsnetze werden im nachfolgenden Abschnitt auszugsweise dargestellt. Die zur Abschätzung möglicher zukünftiger Entwicklungen werden mit entsprechenden Szenarien beaufschlagt, ein solches PV-, WP- und EVCS-Durchdringungsszenario wird ebenso beispielhaft skizziert. Abschließend werden die Simulationsergebnisse anhand entsprechender Grafiken diskutiert.

\subsection{Niederspannungs-Testnetze}

Auszugsweise werden drei der verwendeten NiederspannungsTestnetze im Folgenden beschrieben, wobei der Ausgangszustand jener Zustand ist, bei dem sämtliche relevante Leistungsschalter offen sind

\subsubsection{Modernes, urbanes Niederspannungsnetz (aspern Seestadt, Wien)}

Das Niederspannungsnetz des Stadtentwicklungsgebiets aspern Seestadt (Wien) bietet ein gutes Beispiel für ein neu errichtetes, urbanes Smart Grid. Dieses wird grundsätzlich radial (im offenen Ring) betrieben, verfügt allerdings über eine Vielzahl an Vermaschungsmöglichkeiten respektive offener Trennstellen. Die gesamte Topologie sowie das Netz an sich sind gut dokumentiert und mit einer ausreichenden Anzahl an Messdatenerfassungssystemen ausgestattet, welche ein entsprechendes Monitoring ermöglichen. Aufgrund der beschriebenen Topologie und der damit verbundenen Möglichkeit der Rekonfiguration wird dieses Netz vorzugsweise zur Durchführung realitätsnaher Simulationen bzw. Tests des in den vorangegangenen Kapiteln beschriebenen Algorithmus verwendet. Das Gesamtnetz besteht aus 12 Niederspannungsteilnetzen mit 24 Transformatoren 20/0,4 kV, $15 \mathrm{~km}$ Kabelstrecken und mehr als hundert Privatkunden. Für die ersten Tests des SMM-Algorithmus findet das Teilnetz TS10 (siehe Abb. 3) mit den nachfolgend beschriebenen Eckdaten Anwendung:

- zwei Transformatoren 630 kVA, 20/0,4 kV mit 2 Einspeisungen (Feeder)

- 22 Knoten/Terminals mit 21 Leitungen

- Gesamtkabellänge 1,58 km

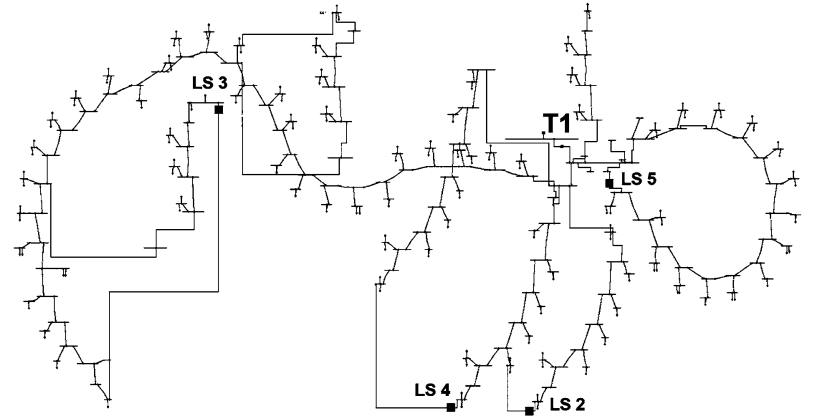

Abb. 4. Rurales Niederspannungstestnetz nach Kerber [13], modifiziert durch AIT samt dem Transformator T1 und den Leistungsschaltern LS 2 bis LS $5[8,10,11]$

- 17 Haushaltskunden

- 4 Leistungsschalter/Trennstellen.

\subsubsection{Rurales Niederspannungsnetz (modifiziertes Netz nach} Kerber)

Abbildung 4 zeigt das ebenso für Testzwecke verwendete rurale Niederspannungsnetzmodell nach Kerber [13] modifiziert durch das AIT. Im Folgenden sind die Eckdaten dieses NS-Netzes zusammengefasst:

- ein Transformator 160 kVA, 10/0,4 kV

- 175 Knoten/Terminals mit 174 Leitungen

- Gesamtleitungslänge 18 km

- Kabelanteil $95 \%$

- 80 Haushaltskunden

- 4 Leistungsschalter/Trennstellen

4.1.3 Synthetisches Niederspannungsnetz Im Folgenden kommt ein synthetisches Niederspannungstestnetz eines Vorprojekts des AIT zum Einsatz (siehe Abb. 5) welches auf realen historischen Messdaten eines europäischen NS-Netzes basiert. Nachfolgend sind dessen Eckdaten zusammengestellt:

- neun Transformatoren 250/400/500 kVA, 20/0,4 kV mit 9 Einspeisungen (Feeder)

- 265 Knoten/Terminals mit 264 Leitungen

- Gesamtkabellänge $26,5 \mathrm{~km}$

- Kabelanteil $45 \%$

- 137 Haushaltskunden, 52 gewerbliche Kunden 


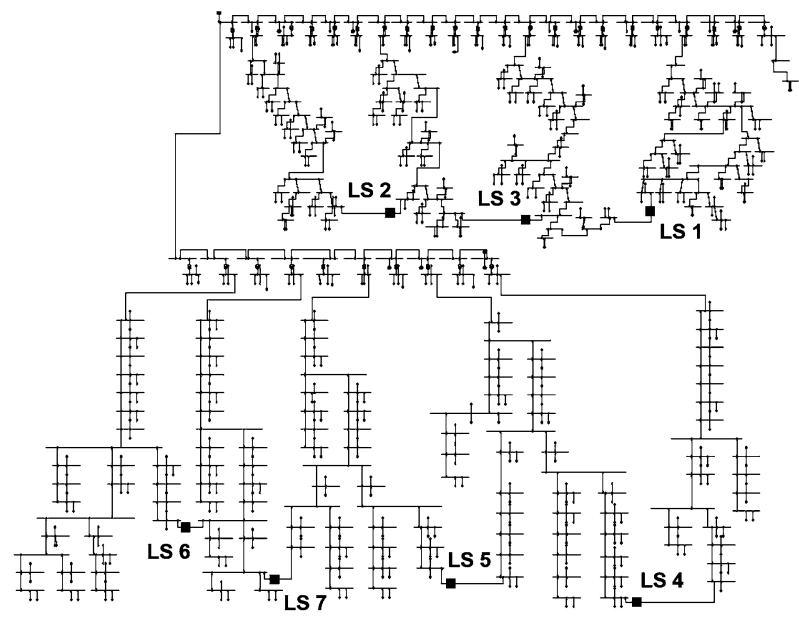

Abb. 5. Synthetisches Niederspannungstestnetz von AIT-Vorprojekt samt Leistungsschaltern LS 1 bis LS $7[8,10,11]$

- 7 Leistungsschalter/Trennstellen.

\subsection{Beispielhafte Durchdringungsszenarien}

Im Zuge der ersten Simulationen und Tests des Algorithmus kommen im Wesentlichen zwei beispielhafte Durchdringungsszenarien zum Einsatz: Einerseits jenes mit und andererseits jenes ohne dezentraler Einspeisung mittels Photovoltaikanlagen (PV-Anlagen). Diese beiden Szenarien sind wie folgt beispielhaft den unterschiedlichen Testnetzen zugeordnet:

- Modernes, urbanes Niederspannungsnetz (aspern Seestadt, Wien) mit historischen Daten gemessen an den Transformatorstationen bzw. an entsprechenden Kabelverteilerschränken

- Szenario A: "ohne PV"

* Haushaltskunden

* 40\% der Lastknoten sind mit Ladestationen für Elektrofahrzeuge beaufschlagt (jeweils $40 \%$ der Knotenlast)

* $40 \%$ der Lastknoten sind mit Wärmepumpen ausgestattet (jeweils 25\% der Knotenlast).

- Szenario B: „mit PV“

* wie Szenario A

* 70\% der Lastknoten sind mit Photovoltaikanlagen ausgestattet (Nennleistung der PV liegt jeweils bei zusätzlich 56\% der Knotenhaushaltlasten; dabei wurde die Nennleistung der PV so angepasst, dass die Betriebsmittel des Netzabschnittes thermisch ausgelastet sind).

- Rurales Niederspannungsnetz mit skalierten, synthetischen Lastprofilen unter Berücksichtigung der Nominallasten gemäß den Daten nach Kerber [13]

- Szenario A: "ohne PV"

* 80 Haushaltskunden

- Szenario B: "mit PV"

* wie Szenario A

* $50 \%$ der Lastknoten sind mit Photovoltaikanlagen ausgestattet.

- Synthetisches Niederspannungsnetz mit skalierten, synthetischen Lastprofilen unter Berücksichtigung von Nominallasten gemäß Daten unterschiedlicher EU-Länder

- Szenario A: "ohne PV"

* 137 Haushaltskunden

* 52 gewerbliche Kunden

- Szenario B: „mit PV”
* wie Szenario A

* 50\% der Lastknoten sind mit Photovoltaikanlagen ausgestattet.

Die für die simulativen Tests erforderlichen Verbraucherlastdaten basieren auf realen Messwerten wobei die benötigten Lastdaten für PV-Anlagen, Ladestationen für Elektrofahrzeuge oder Wärmepumpen hierbei auf den Lastprofilen von SimBench [14] beruhen. $[8,11,12]$

\subsection{Simulationsergebnisse der SMM-Tests}

Die in den vorangegangenen Abschnitten diskutierten Testnetze sowie Szenarien werden in weiterer Folge zur Validierung des entwickelten Algorithmus zur Implementierung des SMM in der zuvor beschriebenen Entwicklungsumgebung verwendet. Dazu werden die Niederspannungstestnetze als digitales Netzmodell in DIgSILENT PowerFactory angelegt und mit möglichen zukünftigen Durchdringungsszenarien hinsichtlich PV-, EVCS- bzw. WärmepumpenAusbau angereichert wobei für jedes Testnetz jeweils ein Szenario mit PV-Integration und eines ohne näher untersucht wird. In den folgenden Unterabschnitten werden die in Abschn. 3.1.3 eingeführten KPIs sowie die damit verbundene Sortierung bzw. Priorisierung der verschiedenen Netzkonfigurationen diskutiert und im Sinne der Vergleichbarkeit anhand von Radar-Charts dargestellt. Hinsichtlich der Beeinflussung der KPIs durch die zugehörigen Gewichtungsfaktoren werden entsprechende Sensitivitätsanalysen durchgeführt und mittels Carpet-Plots veranschaulicht.

\subsubsection{Simulationen zu urbanem Niederspannungsnetz (aspern Seestadt, Wien)}

Die ersten Ergebnisse der simulativen Tests des SMM Algorithmus werden anhand des in Abschn. 4.1.1 vorgestellten NS-Netzmodells aspern Seestadt (Teilnetz TS10) diskutiert. Dabei werden die möglichen Schaltzustände von vier verfügbaren Leistungsschaltern sowie die daraus resultierenden Netzkonfigurationen durch die Berechnung der zuvor beschriebenen KPIs bewertet.

Die folgenden beiden Abbildungen zeigen jeweils im oberen Bereich (a) die verschiedenen sechs KPIs und im unteren Bereich (b) die berechnete Summe der einzelnen KPIs sortiert nach der jeweiligen Priorisierung der Netzkonfigurationen. In Abb. 6 ist dabei das Ergebnis des Szenarios mit Photovoltaik, in Abb. 7 jenes des Szenarios ohne PV dargestellt.

Aufgrund der Tatsache, dass die KPI-Gewichtungsfaktoren $k_{1}$ bis $k_{6}$ für Testzwecke vorerst gleich eins ( $\left.{ }^{\prime \prime}{ }^{\prime \prime}\right)$ gesetzt sind, kann der maximale Wert der Summe aller sechs KPIs (Abb. 6 (b) bzw. 7(b)) einen Maximalwert von sechs $\left({ }^{\prime \prime} 6 "\right)$ erreichen. Gemäß den Definitionen (1) bis (8) aus Abschn. 3.1.3 ist der beste Fall ein niedriger Wert eines KPIs (im Bereich von 0, geringe negative Auswirkungen) und der schlechteste Fall ein hoher Wert eines KPIs (im Bereich von 1 , große negative Auswirkungen).

Tabelle 2 veranschaulicht die Kombinatorik der einzelnen Leistungsschalter und schafft damit eine Zuordnung der einzelnen Schalterstellungen zu den jeweiligen Netzkonfigurationen. Die dabei verwendeten booleschen Werte symbolisieren dabei einen offenen $\left({ }^{\prime \prime} 0^{\prime \prime}\right)$ sowie einen geschlossenen („1“) Leistungsschalter.

Ergebnisdarstellung als Radar-Chart Die Darstellung der Netzkonfigurationen in den nachfolgenden Radar-Charts ist so gewählt, dass jene mit der höchsten Priorität oben (auf "zwölf Uhr") dargestellt wird und alle Weiteren im Uhrzeigersinn mit absteigender Priorisierung. 
KPIs abhängig von der Netzkonfiguration

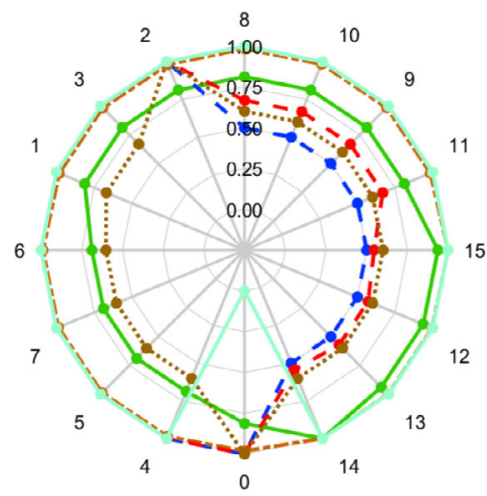

(a)

Summe der KPIs abhängig von der Netzkonfiguration

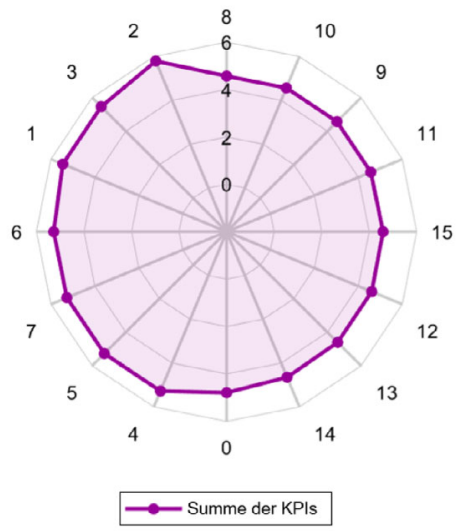

(b)

Abb. 6. KPIs in Abhängigkeit der Netzkonfiguration für das urbane NS-Netz aspern Seestadt (Teilnetz TS10) mit LS 2 bis 5 sortiert nach Priorisierung, Szenario mit PV

Tab. 2. Beispielhafte Zuordnung Netzkonfiguration zu Schaltkombinatorik für aspern Seestadt (Teilnetz TS10)

\begin{tabular}{ccccc}
\hline Netz-konfiguration & \multicolumn{4}{l}{ Leistungsschalter } \\
\cline { 2 - 5 } & LS 2 & LS 3 & LS 4 & LS 5 \\
\hline 0 & 0 & 0 & 0 & 0 \\
1 & 0 & 0 & 0 & 1 \\
2 & 0 & 0 & 1 & 0 \\
3 & 0 & 0 & 1 & 1 \\
4 & 0 & 1 & 0 & 0 \\
5 & 0 & 1 & 0 & 1 \\
6 & 0 & 1 & 1 & 0 \\
7 & 0 & 1 & 1 & 1 \\
8 & 1 & 0 & 0 & 0 \\
9 & 1 & 0 & 0 & 1 \\
10 & 1 & 0 & 1 & 0 \\
11 & 1 & 0 & 1 & 1 \\
12 & 1 & 1 & 0 & 0 \\
13 & 1 & 1 & 0 & 1 \\
14 & 1 & 1 & 1 & 0 \\
15 & 1 & 1 & 1 & 1 \\
\hline
\end{tabular}

KPIs abhängig von der Netzkonfiguration

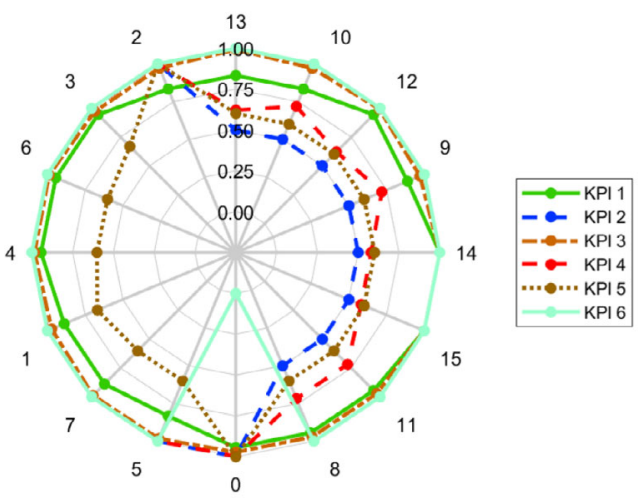

(a)

Summe der KPIs abhängig von der Netzkonfiguration

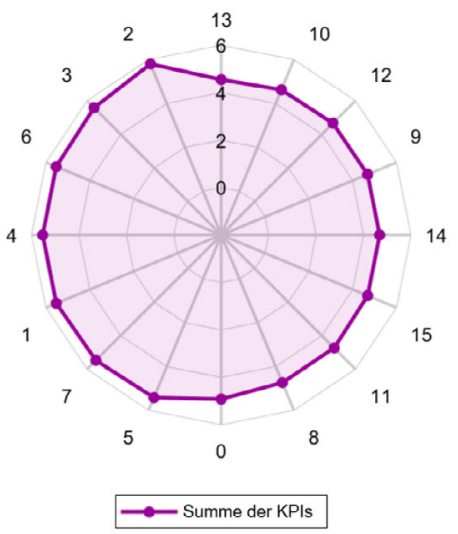

(b)

Abb. 7. KPIs in Abhängigkeit der Netzkonfiguration für das urbane NS-Netz aspern Seestadt (Teilnetz TS10) mit LS 2 bis 5 sortiert nach Priorisierung, Szenario ohne PV

Abbildung 6 (a) zeigt beispielsweise, dass $\mathrm{KPI}_{6}$ (Vermaschung) nur bei der Netzkonfiguration 0 (alle Trennstellen bzw. alle Leistungsschalter offen) zu einem Wert von $\mathrm{KPI}_{6}=0$ führt. Es lässt sich erkennen, dass $\mathrm{KPI}_{2}$ (Verletzung Leitungsauslastung) nur für die Hälfte der Varianten $(8,10,9,11,15,12,13,14)$ im Bereich von 0,5 liegt. Dies bedeutet, dass weniger Verletzungen der Leitungsauslastungen zu erwarten sind als bei den anderen Varianten. Darüber hinaus ist in Abb. 6 (b) die Gesamtsumme der KPIs entsprechend der Priorisierung der verschiedenen Netzkonfigurationen in absteigender Reihenfolge dargestellt. Die Varianten 0 (alle Leistungsschalter offen) und 15 (alle Leistungsschalter geschlossen) sind hinsichtlich der Bewertung des Algorithmus anhand der berechneten KPIs nicht zu bevorzugen. Dies lässt darauf schließen, dass der Rekonfigurationsansatz zu einer Verbesserung der Ausgangssituation führt. Als geeignetster Fall bzw. als optimale Netzkonfiguration ergibt sich demnach die Variante 8 wo nur Leistungsschalter LS 2 (gemäß Abb. 3) geschlossen ist.

Im Vergleich dazu zeigt Abb. 7 (b) eine geänderte Priorisierung aufgrund des verwendeten Szenarios ohne PV (sprich mit einer anderen Lastsituation), wobei die Netzkonfiguration 13 anstelle von 8 als zu bevorzugende ermittelt wird. Auffallend ist auch, dass sowohl beim Szenario "mit PV" als auch bei jenem "ohne PV" Netzkonfiguration 2 als am Schlechtesten bewertet wird. Dies ergibt sich im 

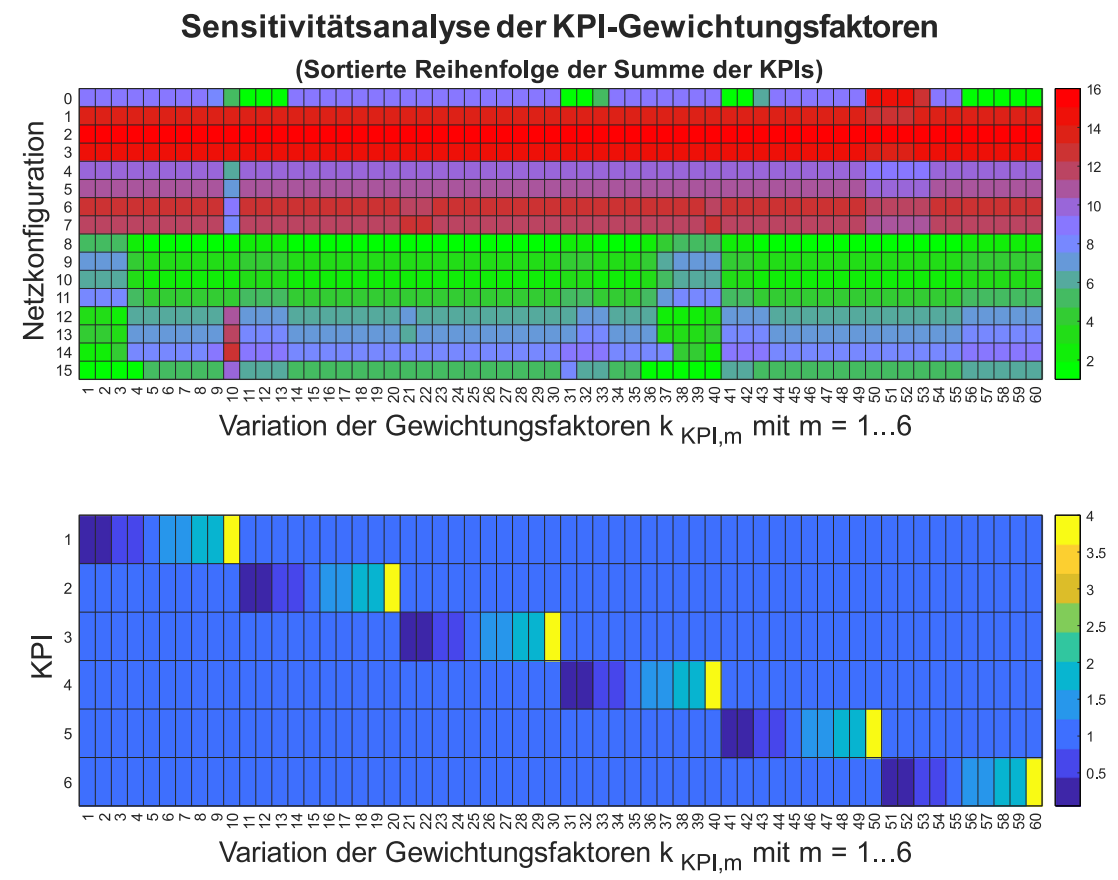

Abb. 8. Sensitivitätsanalyse der KPI-Gewichtungsfaktoren $\mathrm{k}_{\mathrm{KPI}, \mathrm{n}}$ für das urbane NS-Netz aspern Seestadt (Teilnetz TS10), Szenario mit PV

Sensitivitätsanalyse der KPI-Gewichtungsfaktoren

(Sortierte Reihenfolge der Summe der KPIs)
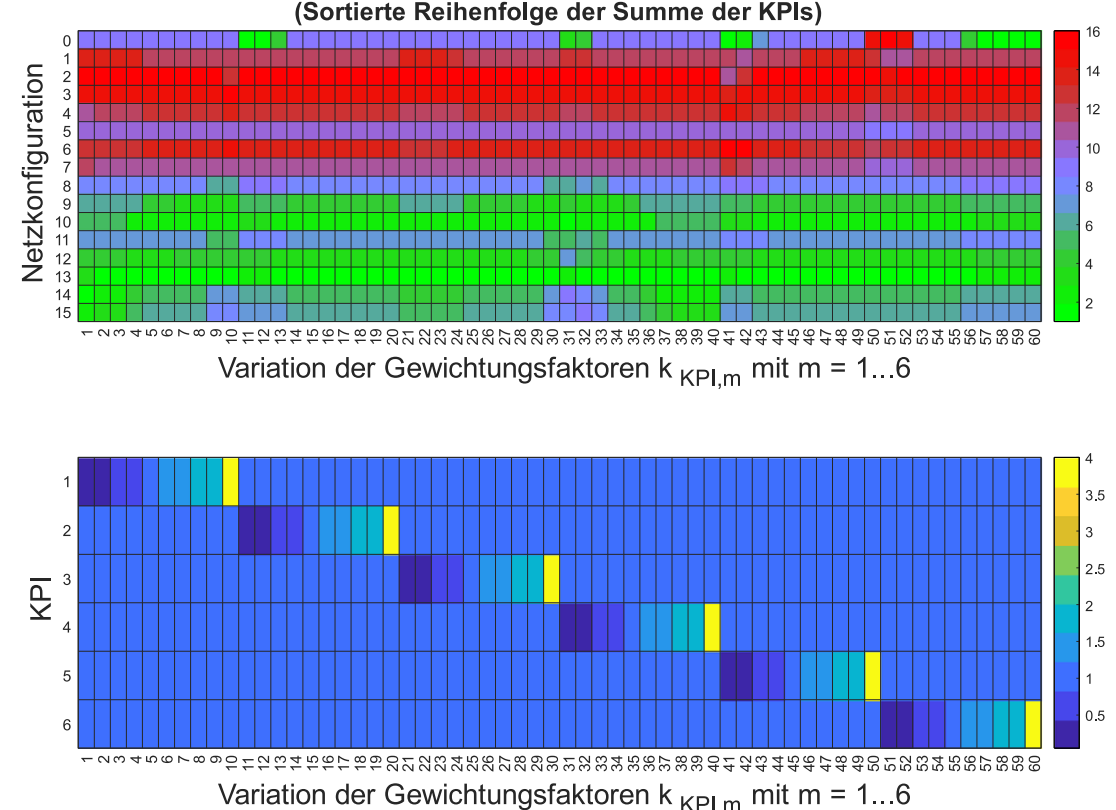

Abb. 9. Sensitivitätsanalyse der KPI-Gewichtungsfaktoren $k_{K P I, n}$ für das urbane NS-Netz aspern Seestadt (Teilnetz TS10), Szenario ohne PV

Wesentlichen aus dem Zusammenspiel der einzelnen sechs KPIs, im Speziellen jedoch hier durch die verhältnismäßig große Entfernung des betroffenen Leistungsschalters (bei Netzkonfiguration 2 handelt es sich dabei gemäß Tab. 2 um LS 4) zur am häufigsten ausgelasteten Leitung, was wiederum $\mathrm{KPI}_{5}$ entspricht.

Sensitivitätsanalyse als Carpet-Plot Als nächster Schritt werden die Gewichtungsfaktoren $k_{\mathrm{KPI}, \mathrm{m}}$ der einzelnen KPIs in insgesamt 10 Schritten variiert (gewählter Bereich: 0,$05 ; 0,25 ; 0,5 ; 0,75 ; 1,0$;
$1,25 ; 1,5 ; 1,75 ; 2,0 ; 4,0)$ um die Sensitivität der ermittelten Priorisierung hinsichtlich der einzelnen Bewertungskriterien zu untersuchen. Um die Anzahl der Variationen deutlich zu reduzieren, wird jeweils nur ein KPI-Gewichtungsfaktor innerhalb des angegebenen Bereichs verändert, alle weiteren werden auf dem Wert 1,0 belassen. Abbildung 8 repräsentiert die Ergebnisse dieser Sensitivitätsanalyse anhand eines Carpet-Plots für das Szenario mit installierter PV. Darin erkennt man im unteren Teil den jeweiligen Wert der, den sechs KPIs zugeordneten, Gewichtungsfaktoren $k_{\mathrm{KPI}, \mathrm{m}}(\mathrm{m}=1 \ldots 6 \mathrm{KPIS}$, 

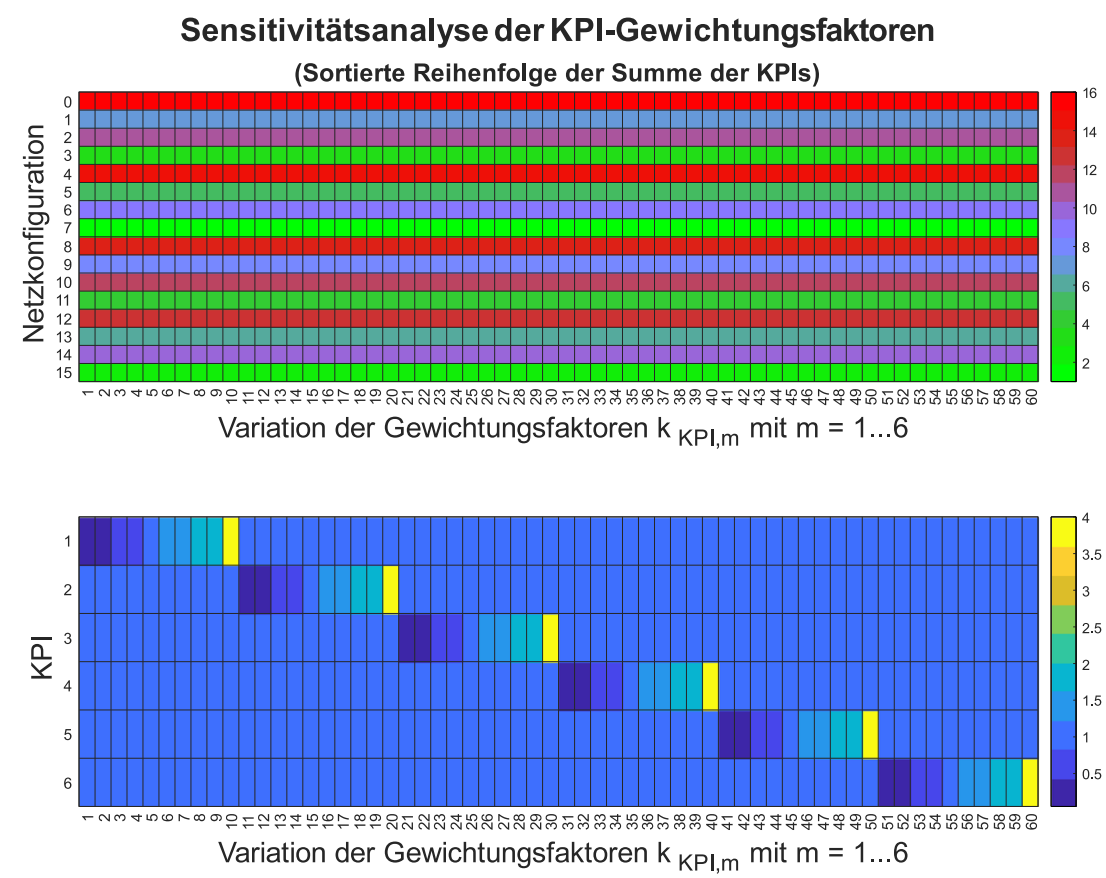

Abb. 10. Sensitivitätsanalyse der KPI-Gewichtungsfaktoren $\mathrm{K}_{\mathrm{KP}, \mathrm{n}}$ für das modifizierte rurale Netz nach Kerber [13], Szenario mit PV

10 Stufen $\cdot 6 \mathrm{KPIS}=60$ Spalten im Plot) in Abhängigkeit der entsprechenden 60 Variationen. Im oberen Teil der Abb. 8 ist die sortierte Reihenfolge der berechneten Summe der KPIs (welche einen Wertebereich von 1 bis $\mathrm{n}=$ Anzahl der möglichen Netzkonfigurationen annehmen kann) zu erkennen, wobei die höchste Priorität durch den Wert 1 symbolisiert wird (hellgrün auf der Farbskala).

Die gewählte Art der Visualisierung stellt die Priorisierung bzw. die Sortierung der einzelnen Netzkonfigurationen bezogen auf die Summe der KPIs dar, was in einer diskreten Verteilung mit insgesamt 16 Werten (= Anzahl der möglichen Netzkonfigurationen pro Variation der Gewichtungsfaktoren) resultiert. Es ist zu erkennen, dass sich grundsätzlich zwei Hemisphären ausbilden: Einerseits jene der Netzkonfigurationen 0 bis 7 im oberen Teil mit einer eher negativen Bewertung (roter Bereich), und andererseits jene der Netzkonfigurationen 8 bis 15 mit einer eher positiven Bewertung (grüner Bereich). Ein Vergleich mit der Zuordnung in Tab. 2 lässt erkennen, dass hierbei das Schließen des Leistungsschalters LS 2 das Verhalten des Netzes positiv beeinflusst. Ebenso ist zu bemerken, dass beispielsweise nahezu alle KPIs bzw. deren Gewichtungsfaktoren Einfluss auf die Netzkonfiguration 0 (alle Leistungsschalter geöffnet) nehmen.

Im Gegensatz dazu wird in Abb. 9 die Sensitivitätsanalyse des beispielhaften Szenarios ohne PV dargestellt.

Es zeigt sich, dass kaum Unterschiede zwischen Abb. 8 (Szenario mit PV) und Abb. 9 (Szenario ohne PV) erkennbar sind. Die Ausnahmen bilden einerseits die untere Hemisphäre (Netzkonfiguration 8 bis 15), welche auf einen differenzierten Einfluss der KPIGewichtungsfaktoren schließen lässt und andererseits einige Unterschiede hinsichtlich der Priorisier-Reihenfolge der Netzkonfigurationen in der Gewichtungsfaktor-Variante 10.

Insgesamt ist festzustellen, dass nur bei sehr kleinen oder sehr großen Gewichtungsfaktoren (z. B. die Gewichtungsfaktoren 0,05 oder 4,0), mit denen die KPIs multipliziert werden, merkliche Einflüsse vorhanden sind. Außerdem ist zu erkennen, dass die Priorisierung einiger Netzkonfigurationen fast völlig unabhängig von unterschiedlichen Gewichtungsfaktoren sowie unterschiedlichen Szenarien ist, z. B. stellt die Konfiguration 2 nahezu immer die schlechteste Variante bzw. jene mit der geringsten Priorisierung dar.

\subsubsection{Simulationen zu ruralem Niederspannungsnetz (modifiziertes Netz nach Kerber)}

Das modifizierte rurale Netzmodell nach Kerber (siehe Abschn. 4.1.2 bzw. [13]) wird als weiteres NS-Testnetz zur simulativen Untersuchung des SMM-Algorithmus verwendet. Wie bereits in Abschn. 4.3.1 beschrieben werden auch hier die möglichen Schaltzustände von vier verfügbaren Leistungsschaltern sowie die daraus resultierenden Netzkonfigurationen (siehe dazu auch die sinngemäß anwendbare Zuordnung gemäß Tab. 2) durch die Berechnung von sechs verschiedenen KPIs bewertet. Diese Evaluierung wird wiederum unter Zuhilfenahme einer Variation unterschiedlicher KPIGewichtungsfaktoren einer entsprechenden Sensitivitätsanalyse unterzogen und hier nur als Carpet-Plot wiedergegeben.

Die folgenden beiden Grafiken zeigen die Ergebnisse der beiden Szenarien mit und ohne PV-Durchdringung. In Abb. 10 (Szenario mit PV) ist dahingehend zu erkennen, dass keinerlei Zusammenhang zwischen der Priorisierung der einzelnen Netzkonfigurationen und den unterschiedlichen Gewichtungsfaktoren besteht. Verglichen mit den Carpet-Plots der Simulationen anhand des aspern Seestadt Netzes (Abb 8 und 9) lassen sich in diesem Fall auch keine zwei Hemisphären erkennen.

Alternativ dazu ist in Abb. 11 das Ergebnis der Sensitivitätsanalyse des Szenarios ohne PV dargestellt. Im Vergleich zu Abb. 10 lassen sich hierbei auch nur geringfügige Abweichungen in der Sortierung erkennen, einzig bei der KPI-Gewichtungsfaktor-Variante 31 stellt sich eine veränderte Reihenfolge der Priorisierung der Netzkonfigurationen ein. Es kann erwähnt werden, dass im Falle beider Szenarien die Netzkonfigurationen 3, 7, 11 und 15 als die Bevorzugten ermittelt werden. Die Gemeinsamkeit der Konfigurationen besteht dabei darin, dass es sich dabei gemäß Tab. 2 um jene handelt, bei welchen die beiden Leistungsschalter LS 4 und LS 5 geschlossen sind. 

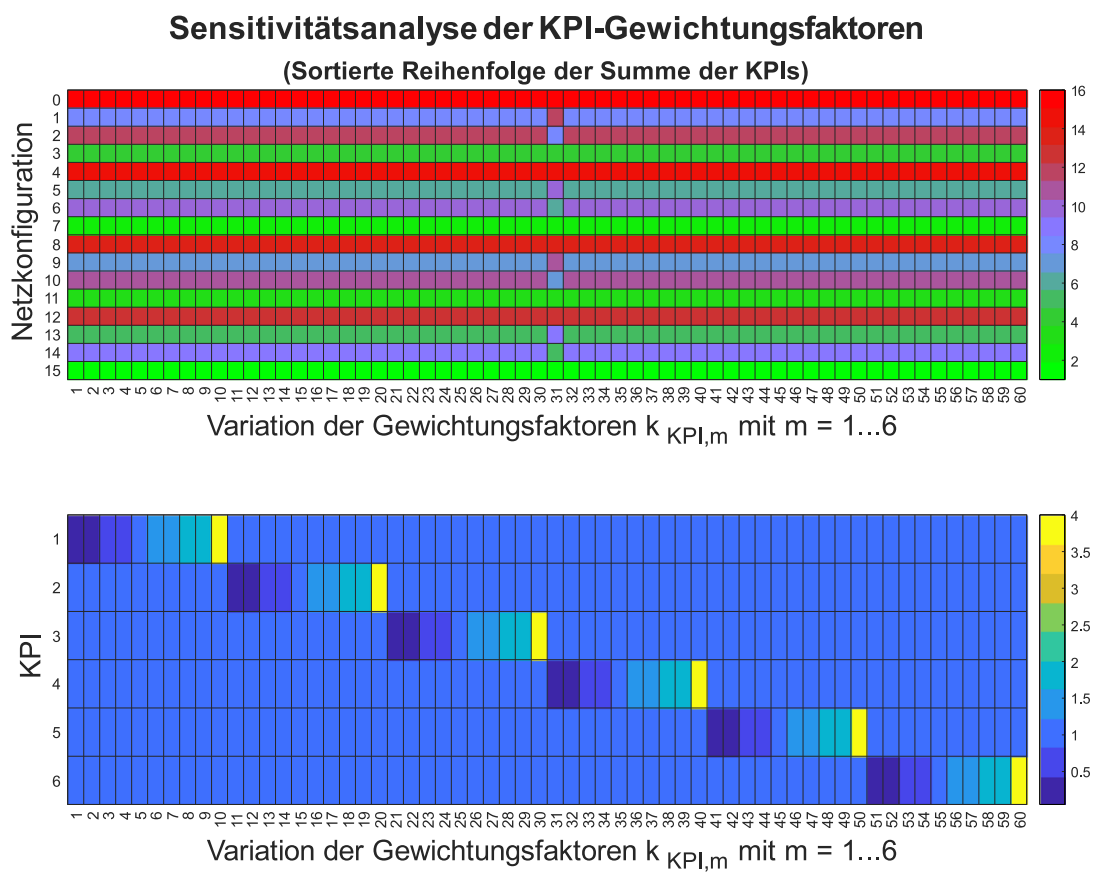

Abb. 11. Sensitivitätsanalyse der KPI-Gewichtungsfaktoren $\mathrm{k}_{\mathrm{KPI}, \mathrm{n}}$ für das modifizierte rurale Netz nach Kerber [13], Szenario ohne PV

Sensitivitätsanalyse der KPI-Gewichtungsfaktoren

(Sortierte Reihenfolge der Summe der KPIs)
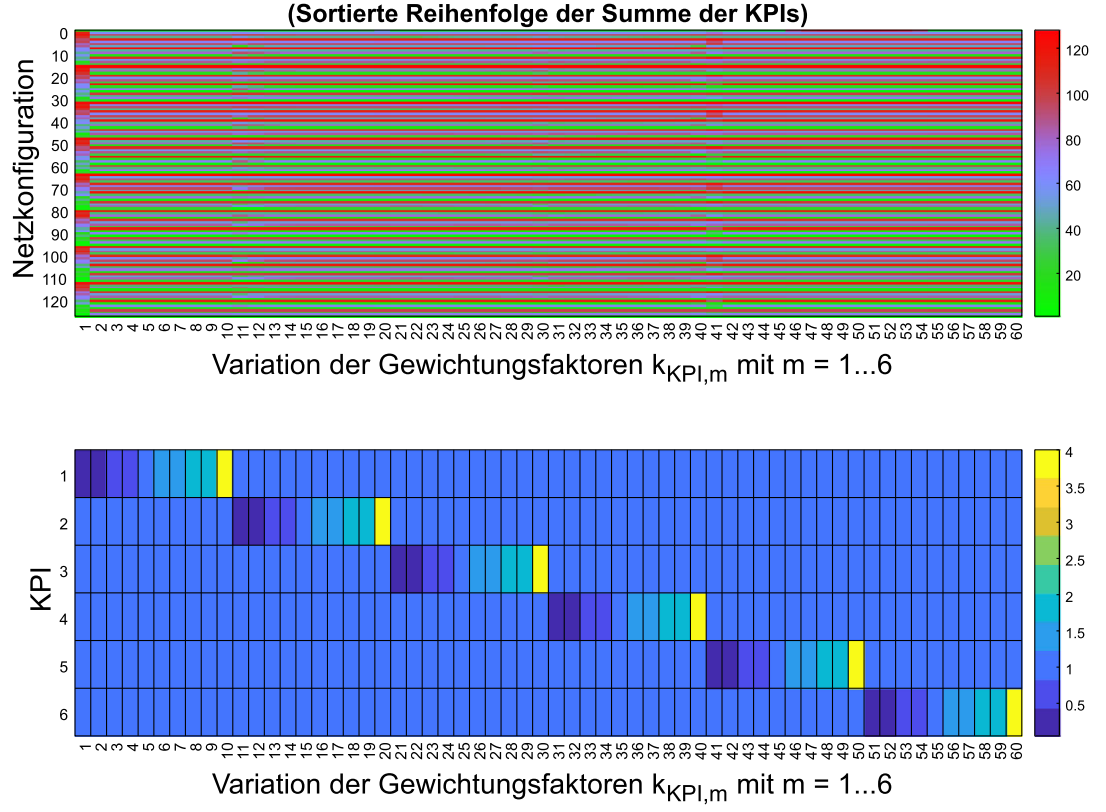

Abb. 12. Sensitivitätsanalyse der KPI-Gewichtungsfaktoren $\mathrm{k}_{\mathrm{KPI}, \mathrm{n}}$ für das modifizierte synthetische Netz, Szenario mit $\mathrm{PV}$

Generell lässt sich am Beispiel des modifizierten NS-Netzes nach Kerber feststellen, dass der Algorithmus hierfür völlig unabhängig von einer entsprechenden Variation der Gewichtungsfaktoren reagiert und stabil dieselben Ergebnisse der Priorisierung der einzelnen Netzkonfigurationen ermittelt.

\subsubsection{Simulationen zu synthetischem Niederspannungsnetz}

Als drittes Beispiel wird das in Abschn. 4.1.3 vorgestellte synthetische Netzmodell einer Analyse mit dem SMM-Algorithmus un- terzogen. Hierbei wird die Priorisierung der, mit Hilfe der sieben Leistungsschalter erreichbaren, Netzkonfigurationen anhand des beschriebenen KPI-Ansatzes untersucht. Abbildung 12 zeigt die Ergebnisse der zugehörigen Sensitivitätsanalyse der unterschiedlichen KPIGewichtungsfaktoren anhand des Szenarios mit PV, Abb. 13 jenes ohne PV.

Verglichen mit den Carpet-Plots der Simulationen anhand des aspern Seestadt Netzes (Abb. 8 und 9) sind im Falle beider Szenarien ebenso keine zwei Hemisphären beobachtbar. Bei detaillierterer 

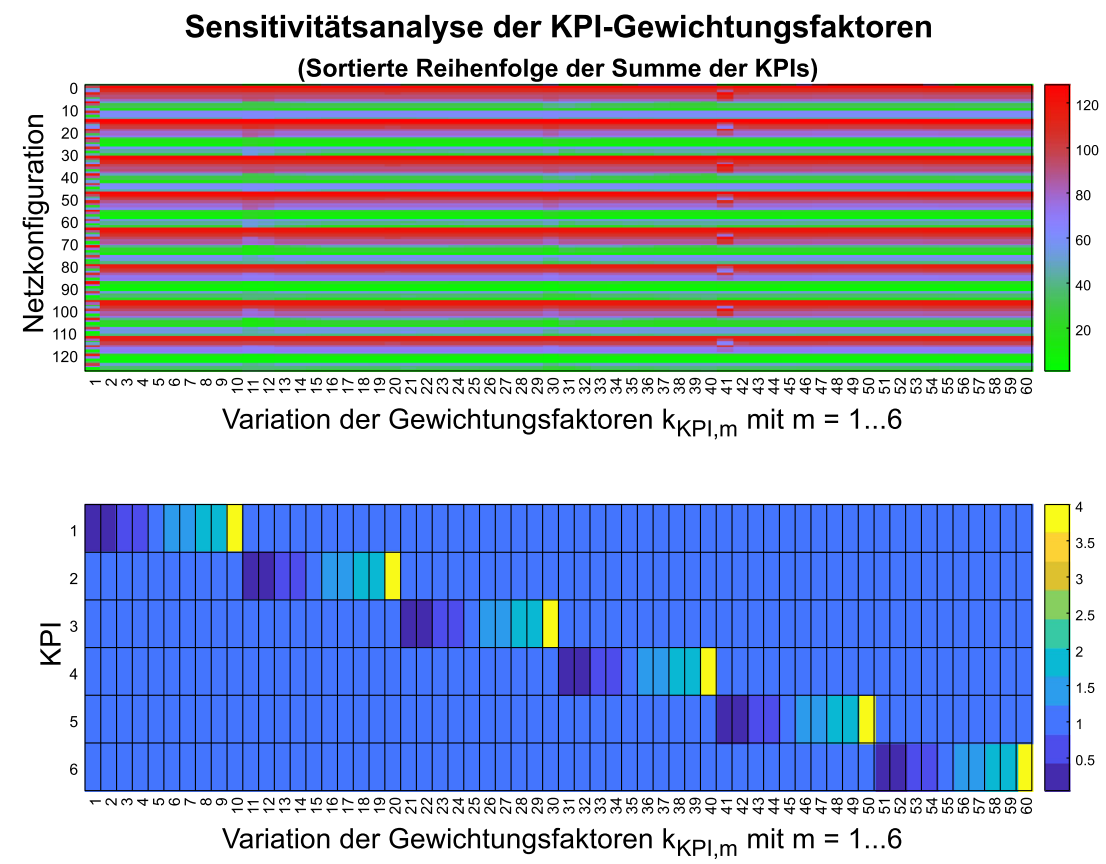

Abb. 13. Sensitivitätsanalyse der KPI-Gewichtungsfaktoren $\mathrm{k}_{\mathrm{KPI}, \mathrm{n}}$ für das modifizierte synthetische Netz, Szenario ohne PV

Betrachtung ist allerdings eine gewisse Abhängigkeit der Priorisierung im Bereich von sehr kleinen Werten der Gewichtungsfaktoren erkennbar, im Speziellen bei $k_{\mathrm{KPI}, 1}, k_{\mathrm{KPI}, 2}$ und $k_{\mathrm{KPI}, 5}$. Auch zeigen sich beim Vergleich der beiden Abb. 12 (Szenario mit PV) und 13 (Szenario ohne PV) deutliche Unterschiede in der Priorisierung der unterschiedlichen Netzkonfigurationen, erkennbar an den vertikalen Schattierungsverläufen in den beiden Grafiken.

Im Allgemeinen lässt sich festhalten, dass auch im Falle des synthetischen Netzmodells (vergleichbar mit dem analysierten Netz nach Kerber in Abschn. 4.3.2) nur geringfügige Abhängigkeiten zwischen der Priorisierung der Netzkonfigurationen und den KPIGewichtungsfaktoren bestehen. Eine mögliche Erklärung hierfür kann beispielsweise die gewählte Variation der Faktoren im Bereich von 0,05 bis 4,0 liefern was eine weiterführende Untersuchung mit einem ausgedehnteren Wertebereich erfordert.

\section{Fazit und Ausblick}

Bezugnehmend auf die zukünftigen Herausforderungen an Niederspannungsnetze (mögliche lokale Überlastungen durch vermehrte Durchdringung von Ladeinfrastruktur für Elektrofahrzeuge, Wärmepumpen, Klimaanlagen oder Photovoltaik-Anlagen) kann der in diesem Beitrag beschriebene Switching Management Module (SMM) Algorithmus mit dessen Ansatz einer automatisierten Rekonfiguration zur Entlastung der Niederspannungs-Verteilnetzinfrastruktur und damit zu einem langfristig stabilen Betrieb dessen beitragen. Die Ergebnisse der softwarebasierten Tests an ausgewählten digitalen Netzmodellen (eingebettet in der dafür konzipierten Entwicklungsumgebung) zeigen ein durchwegs positives Verhalten des entwickelten Algorithmus in punkto Evaluierung der möglichen Netzkonfigurationen. Zu bemerken ist, dass diese bisherigen simulativen Tests mit symmetrischen Lasten bzw. Erzeugern durchgeführt worden sind, eine unsymmetrische Betrachtung des Verhaltens des Algorithmus ist jedoch in Vorbereitung. Es sei erwähnt, dass die Gewichtungsfaktoren in Bezug auf die vorgestellten, zur Priorisierung erforderlichen, Key Performance Indicators (KPIs) durch eine granulare Sensitivitätsanalyse evaluiert bzw. untersucht sowie weiterführend mit unterschiedlichen Verteilnetzbetreibern im Detail diskutiert werden müssen. Ebenso werden noch weitere unterschiedliche, aber vergleichbare Testnetze analysiert, um die Funktionalität des Algorithmus unter Berücksichtigung aller Eventualitäten zu prüfen. Darüber hinaus wird der SMM-Algorithmus im Rahmen weiterer Arbeitspakete des Forschungsprojekts PoSyCo hinsichtlich dessen Einsatzfähigkeit in der projektintern entwickelten Runtime evaluiert und auf einem, speziell dafür designten, Labordemonstrator mit vier Niederspannungs-Kompaktleistungsschaltern praxisnahe getestet.

\section{Danksagung}

Der präsentierte SMM-Algorithmus wurde im Zuge des von der Österreichischen Forschungsförderungsgesellschaft (FFG) und dem österreichischen Klima- und Energiefonds (KLIEN) finanzierten Leitprojekts PoSyCo - Power System Cognification (\#867276) entwickelt.

Funding Note Open access funding provided by Graz University of Technology.

Hinweis des Verlags Der Verlag bleibt in Hinblick auf geografische Zuordnungen und Gebietsbezeichnungen in veröffentlichten Karten und Institutsadressen neutral.

Open Access Dieser Artikel wird unter der Creative Commons Namensnennung 4.0 International Lizenz veröffentlicht, welche die Nutzung, Vervielfältigung, Bearbeitung, Verbreitung und Wiedergabe in jeglichem Medium und Format erlaubt, sofern Sie den/die ursprünglichen Autor(en) und die Quelle ordnungsgemäß nennen, einen Link zur Creative Commons Lizenz beifügen und angeben, ob Änderungen vorgenommen wurden. Die in diesem Artikel enthaltenen Bilder und sonstiges Drittmaterial unterliegen ebenfalls der genannten Creative Commons Lizenz, sofern sich aus der Abbildungslegende nichts anderes ergibt. Sofern das betreffende Material nicht unter der genannten Creative Commons Lizenz steht und die betreffende Handlung nicht nach gesetzlichen Vorschriften erlaubt ist, ist für die oben aufgeführten Weiterverwendungen des Materials die Einwilligung des jeweiligen Rechteinhabers einzuholen. Weitere Details zur Lizenz entnehmen Sie bitte der Lizenzinformation auf http://creativecommons.org/licenses/by/4.0/deed.de. 
Literatur

1. Republic of Austria - Federal Ministry for Sustainability and Tourism, Republic of Austria - Federal Ministry for Transport, Innovation and Technology (2018), \#mission2030 - The Austrian Climate and Energy Strategy.

2. Regulation (EU) 2019/631 of the European Parliament and of the Council setting CO2 emission performance standards for new passenger cars and for new light commercia vehicles, and repealing Regulations (EC) No 443/2009 and (EU) No 510/2011.

3. Directive (EU) 2019/944 of the European Parliament and of the Council on common rules for the internal market for electricity and amending Directive 2012/27/EU.

4. ISO/IEC/IEEE 29148 (2018) ISO/IEC/IEEE International Standard - Systems and software engineering - Life cycle processes - Requirements engineering.

5. Einfalt, A., Brunner, H., Prüggler, W., et al. (2020): In Efficient Utilization of Existing Grid Infrastructure Empowering Smart Communities, IEEE ISGT NA, Washington DC.

6. Projektkonsortium PoSyCo (2020): Deliverable D2.1 - Defined Use Cases and Required Components.

7. Herbst, D., Lagler, M., Schürhuber, R., et al. (2020): In Anforderungen an Niederspannungsnetze und deren Lösungsansätze am Beispiel des Projekts PoSyCo. 16, Symposium Energieinnovation. Graz.

\section{Autoren}

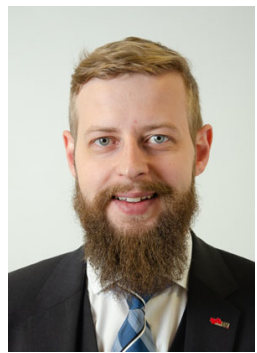

\section{Daniel Herbst}

studierte Elektrotechnik mit Schwerpunkt Energietechnik an der Technischen Universität Graz und war fünf Jahre im Team eines Elektroingenieurbüros in der Obersteiermark in den Bereichen Planung, Ausschreibung und Bauüberwachung tätig. Im Rahmen seiner aktuellen Tätigkeit am Institut für Elektrische Anlagen und Netze der Technischen Universität Graz beschäftigt er sich mit den Themenbereichen Schutzkonzepte im Bereich der Niederspannung, Schutz gegen elektrischen Schlag (Schutzmaßnahmen), Normung, Sicherheit von DC-Ladestationen für Elektrofahrzeuge und Messtechnik in elektrischen Energiesystemen.

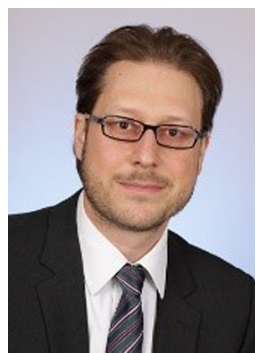

\section{Robert Schürhuber}

Robert Schürhuber promovierte 2003 in Elektrotechnik an der Technischen Universität Wien. Von 2003 bis 2017 arbeitete er in verschiedenen Bereichen der elektrischen Energietechnik für die Firmen Siemens und Andritz Hydro, weiters war er als Lektor im Bereich der elektrischen Energietechnik an der Technischen Universität Wien tätig. Seit Oktober 2017 leitet er das Institut für Elektrische Anlagen und Netze an der Technischen Universität Graz.

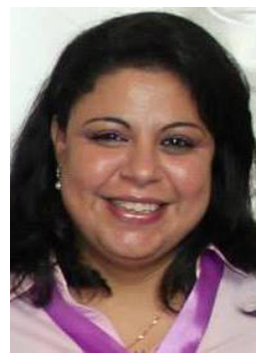

\section{Sawsan Henein}

Sawsan Henein studierte Elektrotechnik mit Schwerpunkt elektrische Energie und Antriebe bzw. Maschinen an den Technischen Universität in Wien und El-Mansoura, Ägypten. Von 1998 bis 2001 arbeitete sie als Forscherin und Laborassistentin an der Technischen Universität El-Mansoura (Ägypten). Seit 2009 ist sie Forscherin am Austrian Institute of Technology im Geschäftsfeld Electric Energy Systems. Sie beschäftigt sich mit den Themen Zuverlässigkeitsbewertung von Stromnetzen, Netzintegration von Erneuerbaren Energien, Netzsimulationen und -optimierung. Ihre Hauptforschungsinteressen sind Zuverlässigkeitsbewertung, Simulation, Anwendung von Steuerungsmethoden von Smart Grids und hybride Energiesysteme.
8. Herbst, D. (laufend) Ein Beitrag zu neuen Ansätzen im Niederspannungsschutz (Arbeitstitel). Dissertation, Technische Universität Graz.

9. Projektkonsortium PoSyCo (2020): Deliverable D3.1 - Report on developed concepts.

10. Herbst, D., Schürhuber, R., Lagler, M., et al. (2021): Low-Voltage Grids in Transition. In Automatic Grid Reconfiguration Approach for Future Smart Grid Challenges, CIRED 2021 Conference, online.

11. Projektkonsortium PoSyCo (2021): Deliverable D3.2 - Mathematical models are developed, tested and reported.

12. Projektkonsortium PoSyCo (2021): Deliverable D3.3 - The interaction of the models in grid model operation is described and reported.

13. Kerber, G. Kerber networks. https://pandapower.readthedocs.io/en/v1.3.0/networks/ kerber.html. Zuletzt abgerufen am 06.07.2021.

14. SimBench - benchmark data set for grid analysis, planning and operation. https:// simbench.de/en/. Zuletzt abgerufen am 06.07.2021.

15. DIgSILENT PowerFactory Software zur Netzberechnung elektrischer Netze. https:// www.digsilent.de/de/powerfactory.html. Zuletzt abgerufen am 10.08.2021.

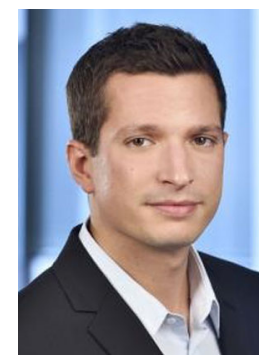

\section{Paul Zehetbaue}

Paul Zehetbauer absolvierte 2014 sein Studium Erneuerbare Energiesysteme an der $\mathrm{FH}$ Technikum Wien. Neben seinem Studium fing er 2013 am Austrian Institute of Technology im Geschäftsfeld Electric Energy Systems als wissenschaftlicher Assistent für diverse Projekte an. Inhaltliche Schwerpunkte umfassen die Themengebiete Netzsimulation und Integration von dezentralen erneuerbaren Energieerzeugern in das elektrische Energiesystem, im Speziellen Photovoltaik. Weitere Beschäftigungsbereiche liegen in den Gebieten Datenanalyse, Entwicklung von Algorithmen, Machine Learning, Forecasting und Grid Codes.

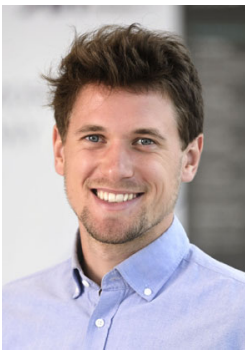

David Fellner

David Fellner absolvierte das Masterstudium Energie- und Automatisierungstechnik an der TU Wien. Er beschäftigte sich in seiner Masterarbeit mit Maßnahmen zur Erhöhung der Spannungsstabilität in schwachen Niederspannungsnetzen samt zugehörigen Simulationen (MATLAB ${ }^{\circledR}$ Simscape ${ }^{\mathrm{TM}}$, DIgSILENT PowerFactory) sowie der Auswertung eines realen Demoaufbaus. Seit 2019 ist er PhDKandidat an der TU Wien und dem AIT. Er forscht zum Thema Monitoring und Fehlererkennung in Stromverteilungsnetzen. Weiters hält er Lehrveranstaltungen über elektrische Stromnetze an der Fachhochschule Technikum Wien.

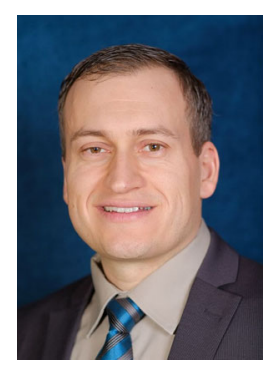

\section{Alfred Einfalt}

Alfred Einfalt ist Principal Key Expert be Siemens AG Österreich in der internen Innovationsabteilung Technology und arbeitet schwerpunktmäßig an der Anwendung von Industrial Internet-of-Things (IloT) basierenden Lösungen im Umfeld von verteilten, dezentralen Energiesystemen. Nach dem Diplomstudium Elektrotechnik an der TU Wien mit Schwerpunkt Energietechnik 2006 begann er als Universitätsassistent am Institut für elektrische Anlagen und Energiewirtschaft an der TU Wien. Neben der Lehrtätigkeit arbeitete er aktiv und auch in koordinierender Rolle an diversen F\&E- 
Projekten im Energieumfeld. Seit Abschluss seines Doktoratsstudiums 2011 arbeitet er als Projektleiter bzw. als technischer Experte in nationalen und internationalen F\&E-Projekten im Bereich Smart Grids bei Siemens AG Österreich.

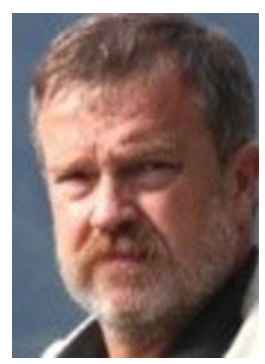

\section{Ernst Schmautzer}

war Senior Scientist am Institut für Elektrische Anlagen und Netze der Technischen Universität Graz und ist nach seiner Pensionierung in beratender Rolle aktiv. Seine Tätigkeiten umfassen: Niederfrequente induktive, ohmsche und kapazitive Beeinflussung, Erdungsund Potentialausgleichssysteme, Schutzmaßnahmen, Normung, niederfrequente elektromagnetische Felder, Elektromobilität, effiziente Nutzung elektrischer Energie, Lastmanagement, intelligente Gebäude, intelligente NS/MS-Netze.

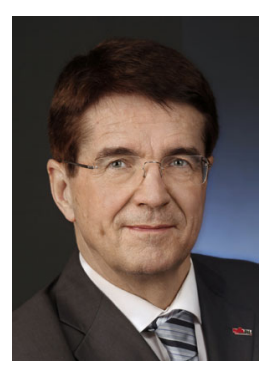

\section{Lothar Fickert}

arbeitete 25 Jahre lang in der Industrie als Schutz- und Anlagentechniker in Netzplanung und Betrieb in der Energiewirtschaft (ELIN UNION, BROWN BOVERI, WIENSTROM). Ab 1998 wirkt er als Professor und Vorstand des Institutes für Elektrische Anlagen und Netze an der Technischen Universität Graz mit den Forschungsschwerpunkten elektrische Energiesysteme, Versorgungssicherheit und Schutztechnik. Als Professor emeritus widmet er sich seit 2017 neben seiner Leidenschaft, der Schutztechnik, auch aktuellen Sternpunktfragen sowie modernen Lehr- und Lernmedien, wo er bisher 100'000 Klicks für seine Schutztechnik-Kurzvideos erhalten hat. 